\title{
Experiential Self-Referential Processing \& Autobiographical Memory Retrieval: A Preliminary Look
}

Kathryn L. Mills

Portland State University

Follow this and additional works at: https://pdxscholar.library.pdx.edu/mcnair Let us know how access to this document benefits you.

\section{Recommended Citation}

Mills, Kathryn L. (2011) "Experiential Self-Referential Processing \& Autobiographical Memory Retrieval: A Preliminary Look," PSU McNair Scholars Online Journal: Vol. 5: Iss. 1, Article 20.

https://doi.org/10.15760/mcnair.2011.190 
Portland State University McNair Research Journal 2011

Experiential Self-Referential Processing \& Autobiographical Memory Retrieval: A Preliminary Look

\author{
by \\ Kathryn L. Mills
}

Faculty Mentor:

Robert Roeser

Citation: Mills, Kathryn L. Experiential self-referential processing \& autobiographical memory retrieval: A preliminary look. Portland State University McNair Scholars Online Journal, Vol. 5, 2011: pages [198-221] 
Experiential Self-Referential Processing \& Autobiographical Memory Retrieval:

A Preliminary Look

Kathryn L. Mills

Mentor: Robert Roeser, PhD

Ronald E. McNair Scholars Program Thesis

Portland State University 


\begin{abstract}
Self-referential processing can be defined as the process of experiencing stimuli as they relate to one’s self (Northoff, 2006). Two distinct modes of self-referential processing, an “experiential mode” of self-referential processing, and a "narrative mode,” have been proposed, and subsequently supported by neuroimaging research (Farb et al., 2007; Gallagher, 2000; Tagini \& Raffone, 2010). Previous studies examining self-reference and memory utilize methods that may only engage a narrative form of self-reference (Macrae et al., 2004; Rogers, Kuiper, \& Kirker, 1977; Symons \& Johnson, 1997), leaving the relationship between the experiential mode of self-reference and memory processes open for investigation. In this study, we examine how mindfulness training may affect (a) the cultivation of mindfulness, a present-oriented, nonjudgmental state similar to the experiential mode of self-reference; (b) executive functions related to mindfulness that make shifting and sustaining awareness in this state possible; (c) the frequency of rumination, a state of self-evaluation similar to the narrative mode of self-reference, and (d) the characteristics of the self-relevant memories one retrieves when asked to during a behavioral task. The study was a 2 x 2 quasi-experimental mixed design with a within-subject (pre-test vs. post-test) and between-subjects factor (mindfulness group vs. control group).
\end{abstract}




\section{Introduction}

Self-referential processing is defined as the process of experiencing stimuli as they relate to one’s self (Northoff, 2006). Two distinct modes of self-referential processing have been proposed, and subsequently supported by neuroimaging research (Farb et al., 2007; Gallagher, 2000; Tagini \& Raffone, 2010). These dual modes include an “experiential mode” of selfreferential processing, characterized by momentary awareness of the psychological present, and a "narrative mode” of self-referential processing involving past experiences and future goals that constitute a key source of representations supporting our continuity of identity over time (Farb et al., 2007; Gallagher, 2000; Tagini \& Raffone, 2010). Although the relationship between selfreference and memory has been explored, these studies utilize methods that may only engage a narrative form of self-reference (Macrae et al., 2004; Rogers, Kuiper, \& Kirker, 1977; Symons \& Johnson, 1997), leaving the relationship between the experiential mode of self-reference and memory processes open for investigation. The present study investigates the relationship between these distinct modes of self-referential processing and behavioral measures of autobiographical memory retrieval and executive functioning in a population of college attending adults receiving mindfulness training.

Our memories provide the foundation for our sense of self, and are often retrieved and adapted to fit with the conception and goals of our current self (Conway \& Pleydell-Pearce, 2000). The memories that serve our sense of self are called autobiographical memories, and the self that is created from these memories is referred to as our autobiographical self. Conway (2005) proposed a Self-Memory System (SMS), composed of a working self that holds an individual's set of active goals and associated self-images, an individual's subjective memories, and the reciprocal relationship between the two. This theory maps onto William James' theory of 
the self being composed of two separate components, the $I$ and $m e$, in which one aspect of the self encompasses the collected experiences of the individual (me), and the other is characterized as the present-moment self ( $I$ ) that experiences (me) (James, 1890). In Conway’s model, the working self is both constrained by one's autobiographical knowledge, and modulating of what autobiographical knowledge is maintained and retrieved (2005). Conway (2005) argues that having a memory system that is conceptually organized and is automatically brought to bear on one's current goals and contexts when relevant is an evolutionary adaptation that has allowed humans to work efficiently and often automatically on tasks in the present moment, and to effectively plan and pursue goals over the longer term.

Aspects of autobiographical memory retrieval can tell us about different aspects of selfprocessing, including the influence of motivations in self-evaluation (e.g. self-enhancement, selfverification), as well as the roles of affect and cognition in these processes (D'Argembeau, Comblain, \& Van der Linden, 2005; D’Argembeau \& Van der Linden, 2008; Sutin \& Robins, 2008). Previous studies have measured how memory retrieval can give insight into our selfreferential processes in which we refer to our autobiographical memories to direct our future behaviors (Williams et al., 2007). The Autobiographical Memory Test (AMT) is a measure that allows us to measure autobiographical memory retrieval processes (Williams \& Broadbent, 1986). The AMT is a primarily a task that measures autobiographical memory specificity, however, other studies have explored other aspects of these retrieved memories with supplemental questions about the vividness and vantage perspective (Kuyken \& Moulds, 2009). Memories can be retrieved from a first-person "field" perspective, where the retriever is looking at the memory through their own eyes, or from a third-person "observer" perspective, where the retriever is looking at the memory from a disembodied observer's perspective (Nigro 
\& Neisser, 1983). The vantage perspective in autobiographical memory retrieval has been hypothesized to reflect motivations underlying self-referential processes (Sutin \& Robins, 2008). The narrative mode as "default"

Research has revealed that in the absence of the demand to attend to external stimuli, humans automatically engage in what is considered the narrative mode of self-referential processing - they retrieve memories about self and fantasize about future selves (McKeirnan et al., 2006). This suggests that a stream of self-relevant mental images, beliefs, and feelings are running rather constantly as a means of non-consciously interpreting "reality” and organizing and directing behavioral responses to that perceived reality. Nonetheless, moments of new learning, creativity, and social intimacy all require a present-oriented state of mind in which past and future are temporarily suspended in favor of the new possibilities of the present moment (Roeser, 2010, personal communication). Thus, shifting from the narrative mode, which is our default mode, into the more present-centered mode, requires training. Mindfulness training, in which one develops an ability to concentrate on and clarify one's present-moment experience, is an ideal means to induce engagement in a more experiential mode of self-referential processing and the benefits it may hold for learning, living, and loving (Roeser, 2010, personal communication). In addition, by definition, such training would be expected to reduce unconstructive narrative modes of self-reference such as rumination by offering an alternative (e.g., returning to the present moment). This study utilizes mindfulness training as a means of inducing participants into engaging more frequently in an experiential mode of self-referential processing. We utilize a self-report measure of rumination and a self-report measure of mindfulness to assess engagement in the narrative and experiential modes of self-referential processing, respectively. 
Moment-to-moment, non-judgmental awareness of experience is the definition of both the experiential mode of self-referential processing, as well as mindfulness, making mindfulness training the ideal means to induce engagement in a more experiential mode of self-referential processing. Indeed, distinct neural correlates of the two proposed modes of self-referential processing were more pronounced in individuals who had received mindfulness training (Farb et al., 2007). Mindfulness practice is thought to lead to a dissociation of the two modes of selfreference, with hypothesized benefits for learning and well being when narrative processes become ruminative, pessimistic, and overly general (Robert Roeser, personal communication, 2010). Furthermore, a previous study found that mindfulness training changes the specificity of retrieved autobiographical memory, possibly by increasing cognitive flexibility (Heeren, Van Broeck, \& Philippot, 2009). Thus, another way mindfulness training, through its cultivation of a present-centered, flexible, and controlled form of awareness, may affect self-referential processing is by making the retrieval of specific (rather than overly general) self-memories more likely thinking about oneself (see Figure 1 for graphical illustration). This study utilizes mindfulness training as a means of inducing participants into engaging in the experiential mode of self-referential processing. We utilize a self-report measure of rumination and a self-report measure of mindfulness to assess engagement in the narrative and experiential modes of selfreferential processing, respectively. 


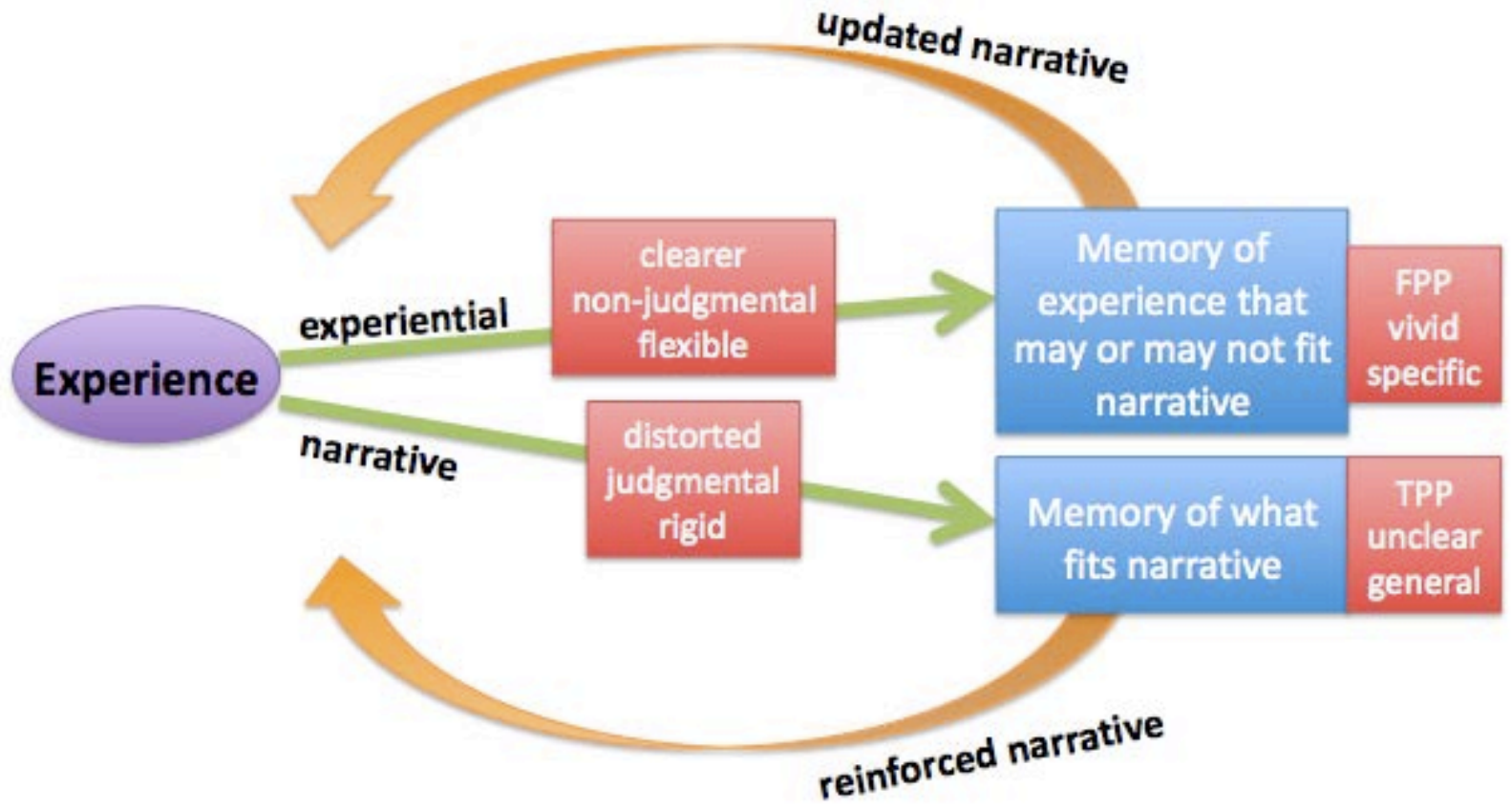

Figure 1: Model illustrates theory of how one's mode of self-referential processing can affect one's memories and self-narrative. Any given experience can be processed through an experiential or narrative mode of selfprocessing. The experiential mode of self-processing is clearer, non judgmental, and flexible, creating a memory that may or may not fit one's self narrative. The narrative mode of self-processing is distorted, judgmental, and rigid, creating a memory that often fits one's narrative. Memories created while in the experiential mode of self-processing are theorized to be recalled from the first person perspective (FPP), as more vivid, and as more specific, while memories created while in the narrative mode of self-processing are theorized to be recalled from the third person perspective (TPP), as less vivid (unclear), and less specific. The newly created memories are then incorporated back into one's self- narrative, either updating the narrative, or reinforcing the narrative.

This present study examines the effects of mindfulness training on young adults’ mindfulness, related executive functioning, and autobiographical memory retrieval using a pre/post-test control-group design. Our theory predicts that mindfulness training will cultivate (a) the practitioner's frequency of engaging in the experiential mode of self-processing (i.e., 
mindfulness), (a) related abilities regarding cognitive flexibility and working memory, and (c) characteristics of the autobiographical memories retrieved during an experimental task.

\section{Theory of Change}

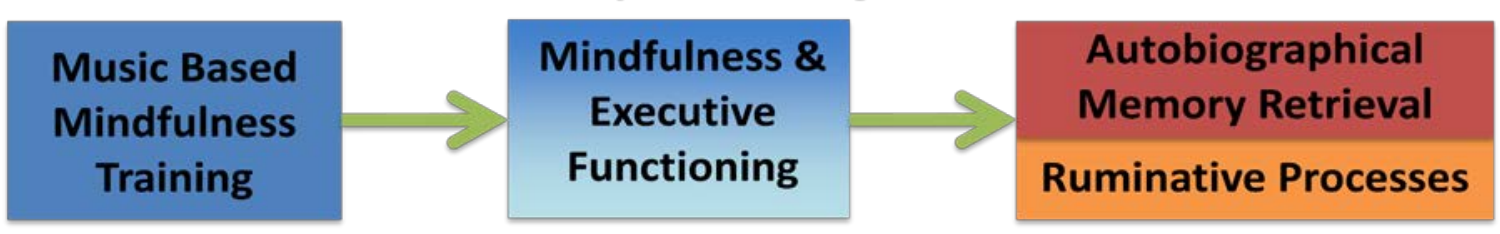

Figure 2: Theory of Change Model. The mindfulness training will increase mindfulness and executive functioning in the students receiving the intervention. This increase in mindfulness and executive functioning will change aspects of autobiographical memory retrieval, presumably increasing the vividness of the memories retrieved, increasing the amount of memories recalled from the first person vantage perspective, and reducing the amount of memories recalled from the third person vantage perspective. The increase in mindfulness and executive functioning will also reduce ruminative processes.

The specific hypotheses addressed in the present study include that the experimental group will (1) increase in self-reported mindfulness at post-assessment; (2) decrease in selfreported rumination at post-assessment; (3) increase in working memory capacity at postassessment; (4) increase in cognitive flexibility at post-assessment; (5) recall more specific, first person perspective, and vivid autobiographical memories at post-assessment. (6) In relation to the mindfulness group only, increased mindfulness will correlate with decreased rumination. (7) The change in autobiographical memories predicted above will correlate with strengthened executive functions and increased mindfulness.

\section{Methods}

Participants

The study was a 2 x 2 quasi-experimental mixed design with a within-subject (pre-test vs. post-test) and between-subjects factor (mindfulness group vs. control group). Those in the 
mindfulness group were recruited from a class offered by the psychology department at Portland State University titled "Contemplative Education and Psychology.” The control group was recruited from another class offered by the psychology department titled "Human Development."

Research assistants visited the classrooms in the first week of spring term to recruit participants for the study. Those enrolled in the Contemplative Education class were offered extra credit for their participation, whereas students in the Human Development class were offered monetary compensation for their participation in each data collection time point. In an attempt to control for inherent bias, control group participants were included only if they expressed interest in enrolling in a class on Contemplative Education on a pre-screen enrollment form. To control for age effects, anyone over the age of 33 was excluded from the analyses.

Sixteen students were recruited for the mindfulness group, and ten students were recruited for the control group. Three students from the mindfulness group, and two control participants, were excluded in the analyses because they did not complete the post-assessment. Demographic characteristics for the mindfulness group ( $\mathrm{n}=13)$ and control group $(\mathrm{n}=8)$ are displayed in Figure 2. There were no significant ( $p>$.49) differences in age between groups.

Figure 3: Demographic Characteristics

\begin{tabular}{lll|ll}
\multicolumn{3}{c}{ Mindfulness Group (n=13) } & \multicolumn{2}{l}{ Control Group (n=8) } \\
\hline \multirow{2}{*}{ Age } & Mean & Std. Dev. & Mean & Std. Dev. \\
\cline { 2 - 5 } & 26.85 & 4.00 & 25.5 & 4.38 \\
\hline Gender & $\%$ & $\mathrm{~N}$ & $\%$ & $\mathrm{~N}$ \\
\hline \multirow{2}{*}{ Female } & $54 \%$ & 7 & $63 \%$ & 5 \\
Male & $46 \%$ & 6 & $38 \%$ & 3 \\
\hline
\end{tabular}

\section{Intervention}

Students enrolled in the Contemplative Education class received and 8-week, 16-hour mindfulness intervention by a trained facilitator. The mindfulness intervention used in this 
study, the Music-Based Mindfulness Training (MBMT) program, was developed by Shinzen Young as a program was designed to teach college students basic mindfulness skills and their application as coping techniques for everyday stressors.

\section{Assessment}

During the first two weeks of the academic quarter, and again during the last two weeks of the academic quarter, each group completed a battery of validated behavioral measures and self-report questionnaires. Each participant was administered the battery of behavioral measures alone in a quiet room by a trained research assistant. The assessment itself lasted 90-120 minutes, and consisted of five behavioral measures. The Automated Operation Span Task, Sustained Attention to Response Task, Autobiographical Memory Test, and Michigan Fish Task were all administered on standardized laptop computers. The Five Point Test was administered by hand. This study concerns the results from three behavioral measures: the Automated Operation Span Task, Five Point Test, and Autobiographical Memory Test.

Students completed a self-report survey. Surveys were sent participants through a secure online survey program. The self-report questionnaires measures used included the Attentional Control Scale, Five Facet Mindfulness Scale, Rumination-Reflection Questionnaire, SelfTheories of Intelligence, Self-Construal Scale, Self-Compassion Scale, Achievement Goals Scale, Positive and Negative Affectivity Scale and Self-Forgiveness Scale. The self-report questionnaire measures used in this study included the Five Facet Mindfulness Scale and the Rumination-Reflection Questionnaire.

Measures

Participants completed the Automated Operation Span Task (OSPAN: Unsworth et al., 2005), a validated measure of an individual's working memory capacity. The OSPAN asks 
participants to switch back and forth between cognitive processes while at the same time holding certain objects in working memory. Participants are instructed to complete mathematical operations and memorize words in alternation (Turner \& Engle, 1989). Individuals who perform well on this task are thought to have more limited-capacity attentional resources available to them and therefore are better able to perform tasks requiring sustained, voluntary attention (e.g., Conway \& Engle, 1996).

The Five Point Test measures figural fluency in each participant (Regard et al., 1982). Participants are given three minutes to draw as many different figures as possible on a sheet of paper with fifty identical rectangles that contain five dots. This measure was used based on the suggestion of previous studies to use a non-verbal fluency to task to measure cognitive flexibility (Heeren at al., 2009).

To assess characteristics of retrieved autobiographical memories, the study utilized a modified version of the Autobiographical Memory Test (AMT: Williams \& Broadbent, 1986), formatted for administration on a laptop. Participants were instructed to retrieve specific memories of events that had happened in their life, excluding memories of events that had happened in the last week. In our study nine cue words, divided into equal groups of positive, negative, and neutral words, were presented in alteration to each participant on a computer screen above an empty text box, with the instructions: "For each word we want you to think of an event that happened to you that the word reminds you of.” Participants were given 30 seconds to retrieve a memory and type it into the text box provided on the screen. A practice trial was given before the nine cue words were administered. Difference cue words were used for each assessment period. After recalling the nine cued autobiographical memories, each memory reappeared on the screen in the same order, with instructions to answer a few questions 
regarding the memory presented on the screen. Participants were asked to rate the vividness of their memory from 1 (No Image) to 5 (As if I were seeing it now). Participants were also asked how many times have you thought about this memory since it has occurred, with the possible answers: Never thought about it until now, 2-5 times, 6-10 times, 11-20 times, 20-100 times, or more than 100 times. Participants were given a blank text box to fill in how old they were when the event occurred. Participants were also asked to rate how important the memory was for them, from 1 (Not at all important) to 5 (Very important). To measure vantage perspective, participants were asked, “did you recall this memory from a first person perspective (i.e., seeing scene through your own eyes) or a third-person perspective (seeing the scene from an observer's perspective)?” They were given the following choices as answers: completely through my own eyes and body, mostly through my own eyes and body, equally through my own eyes and body and as an observer, mostly as an observer, completely as an observer, and I'm not sure.

Memories not omitted were later coded as specific, categorical, or extended. A memory was considered omitted if the text field was blank, or the memory was of an event that had occurred within a week of the assessment. Two independent raters were used to rate 27 memories, to establish interrater reliability $(\mathrm{k}=.98)$, after which only one rater was used. For the purposes of this study, we only analyzed memory specificity, vividness, and vantage perspective.

The Five Facet Mindfulness Questionnaire (FFMQ: Baer et al., 2006) was used to assess self-reported mindfulness. The FFMQ is a scale of 39 items that was developed to be representative of five distinct facets of mindfulness: observing, describing, acting with awareness, non-judging of inner experience, and non-reactivity to inner experience (Baer et al., 2006). Participants were given the following instructions: "Listed below are a number of 
statements. Read each one as if it referred to you. Please rate each of the following statements using the scale provided. Please respond to every statement.” Participants were asked to rate their response on a scale ranging from 1 (Never) to 5 (Always).

The Rumination-Reflection Questionnaire (FFQ: Trapnell \& Campell, 1999), was used to assess ruminative self-focus in participants. The RRQ was designed to distinguish reflective vs. ruminative self-focused behavior by asking participants to rate their agreement with 24 items (Trapnell \& Campell, 1999). For this study, participants were given the following instructions: “Listed below are a number of statements. Read each one as if it referred to you. Beside each statement select the number that best matches your agreement or disagreement. Please respond to every statement,” and instructed to rate their agreement from 1 (Strongly Disagree) to 6 (Strongly Agree).

\section{Analyses Plans}

We conducted three types of analyses. First, we used cross-tabulations to examine sex differences in groups. Second, we used repeated measures analyses of variance to assess time by group change in our dependent variables. Third, we examine correlations between our key mediating and outcome variables.

\section{Results}

Group differences. A 2 by 2 (condition by gender) cross-tabulation was conducted to examine if the groups differed in their gender composition. No significant sex differences by group were found $\left(\chi^{2}(1,20)=.04, p=.85\right)$.

A series of repeated measures analysis of variance models (ANOVAs) were run for each outcome variable in our Theory of Change Model (see Figure 2) with condition as the between- 
subjects factor (treatment vs. matched control) and time (pre-test vs. post-test) as the withinsubjects factor. The existence of a condition by time interaction effect is used as a criterion for program efficacy. Effect sizes in the form of partial eta-squares are reported. Further t-test comparisons were performed to establish any significant $(p<.05)$ differences in means between groups and across time.

Mindfulness. Results of the repeated measures ANOVA for students' self-reported mindfulness as the outcome revealed no significant effect for condition by time (Wilks' $L=.87, F(1,17)=$ 2.56, $p=.13$, eta-squared $=.13$ ). Although there was a marginally significant effect by time (Wilks' $\mathrm{L}=.82, F(1,17)=3.81, p=.07$, eta-squared $=.18$ ), the graph below shows that despite all students showing improvement over time on mindfulness, such improvement was more in evidence among those in the treatment group as predicted. There was no main effect by condition $(\mathrm{F}(1,17)=0.10, p=.76$, eta-squared $=.01)$. Mindfulness showed no significant difference

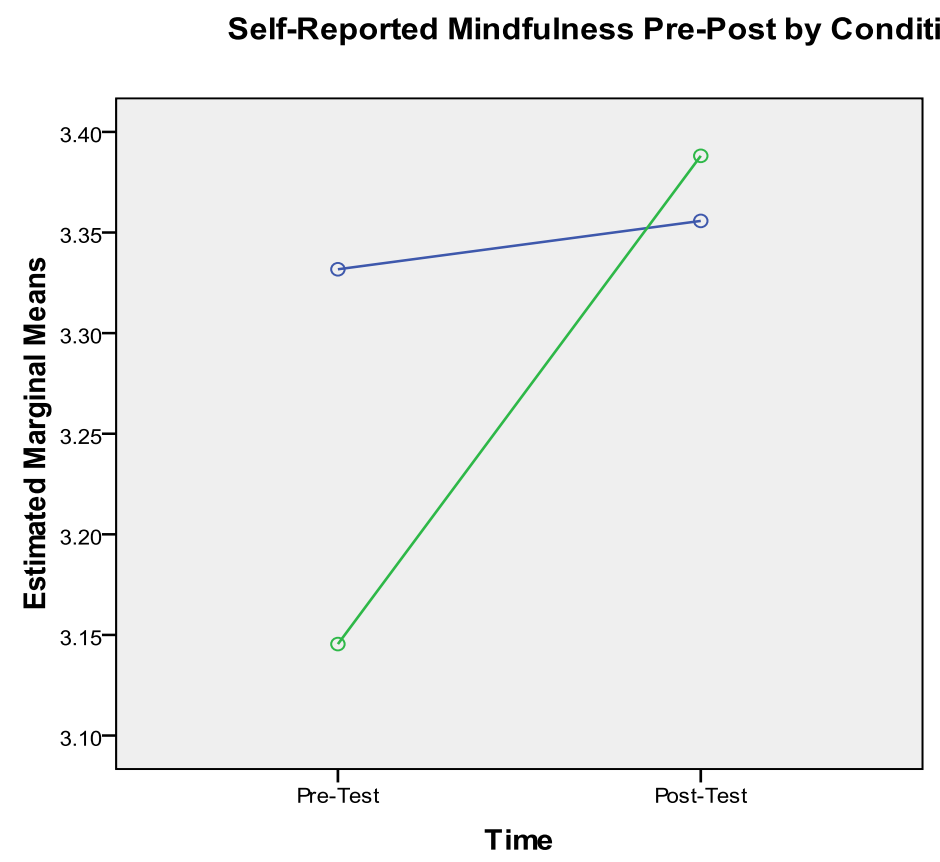
between the experimental group

$\begin{array}{ll}\begin{array}{c}\text { Condition } \\ \text { — Matched Control } \\ \text { Experimental }\end{array} & (M=3.18, S D=.43) \text { and } \\ & \text { control group }(M=3.33, \\ & S D=.62) \text { at pre- } \\ & \text { assessment }(p>.54) . \\ & \text { Post-assessment } \\ & \text { measurement indicated a } \\ & \text { significant increase in } \\ & \text { self-reported }\end{array}$

mindfulness in the experimental group ( $M=3.41, S D=.47, p<.005)$, but no significant increase in 
the control group ( $M=3.36, S D=.66, p>.41$ ). There was no significant between group difference in self-reported mindfulness at post-assessment ( $p>.85)$.

Rumination. Results of the repeated measures ANOVA for students' self-reported rumination as the outcome revealed no significant effect for condition by time (Wilks' $\mathrm{L}=.96, F(1,17)=.66, p$ $=.43$, eta-squared $=.04)$. There was a significant effect by time $($ Wilks' $L=.57, F(1,17)=12.78$, $p=.002$, eta-squared $=.43)$. There was no main effect by condition $(\mathrm{F}(1,17)=0.11, p=.74$, etasquared $=.01$ ). Self-reported rumination, showed no significant difference between the experimental group $(M=4.02, S D=1.05)$ and control group $(M=3.84, S D=.81)$ at pre-assessment (p>.67). Post-assessment measurement indicated a significant decrease in self-reported rumination in the experimental group ( $M=3.51, S D=.75, p<.005)$, as well as in the control group $(M=3.50, S D=.88, p<.05)$. There was no significant between group difference in self-reported rumination at post-assessment $(p>.97)$.

Cognitive Flexibility. Results of the repeated measures ANOVA for students' cognitive flexibility as the outcome revealed no significant effect for condition by time (Wilks’ $\mathrm{L}=.97$, $F(1,17)=.59, p=.46$, eta-squared $=.03)$. Time did show a significant effect (Wilks’ $\mathrm{L}=.22$, $F(1,17)=59.53, p<.001$, eta-squared $=.78)$. There was no main effect by condition $(F(1,17)=$ $0.001, p=.98$, eta-squared $<.001)$. Cognitive flexibility showed no significant difference between the experimental group ( $M=34.69, S D=8.13)$ and control group $(M=35.13, S D=8.77)$ at pre-assessment $(p>.91)$. Post-assessment measurement indicated a significant increase in cognitive flexibility in the experimental group ( $M=42.15, S D=8.06, p<.001)$, as well as in the control group ( $M=43.00, S D=12.13, p<.001)$. There was no significant between group difference in cognitive flexibility at post-assessment ( $p>.86)$. 
Working Memory Capacity. Results of the repeated measures ANOVA for students' OSPAN total score as the outcome revealed no significant effect for condition by time (Wilks' $\mathrm{L}=1.0$, $F(1,18)=.08, p=.79$, eta-squared $=.004)$. There was no significant effect by time (Wilks' $\mathrm{L}=$ $.94, F(1,18)=1.14, p=.30$, eta-squared $=.06)$. There was no main effect by condition $(F(1,17)=$ $1.39, p=.25$, eta-squared $=.07$ ). Working memory capacity, as measured by the OSPAN total score, showed no significant difference between the experimental group $(M=34.67, S D=22.29)$ and control group ( $M=43.13, S D=19.80)$ at pre-assessment $(p>.38)$. Post-assessment measurement did not show the hypothesized significant increase in working memory capacity in the experimental group ( $M=38.17, S D=18.44, p>.21)$, nor was any difference found in the control group ( $M=45.25, S D=19.67, p>.37)$. There was no significant between group difference in working memory capacity at post-assessment ( $p>.43)$.

Specific Autobiographical Memories. Results of the repeated measures ANOVA for students' retrieved specific autobiographical memories as the outcome revealed no significant effect for condition by time (Wilks' $\mathrm{L}=.99, F(1,19)=.16, p=.69$, eta-squared $=.01)$. There was a marginally significant effect by time (Wilks' $\mathrm{L}=.82, F(1,19)=4.18, p=.06$, eta-squared $=.18$ ). There was no main effect by condition $(\mathrm{F}(1,19)=0.74, p=.40$, eta-squared $=.04)$. The number of specific memories retrieved across nine trials of the AMT showed no significant difference between the experimental group $(M=4.69, S D=2.72)$ and control group $(M=5.63, S D=1.85)$ at pre-assessment ( $p>$.36). Post-assessment measurement showed a significant increase in specific memory retrieval in the control group ( $M=7.00, S D=2.07, p<.05)$, but no significant increase was found for the experimental group $(M=5.46, S D=2.47, p<.15)$. There was no significant between group difference in specific memory retrieval at post-assessment ( $p>.14)$. 
Specific Autobiographical Memories recalled from the First Person Perspective. Results of the repeated measures ANOVA for students' specific memories recalled from the first person perspective as the outcome revealed no significant effect for condition by time (Wilks' $\mathrm{L}=1.0$, $F(1,19)=.004, p=.95$, eta-squared <.001). There was not a significant effect by time (Wilks' L $=.98, F(1,19)=.41, p=.53$, eta-squared $=.02)$. There was a main effect by condition $(F(1,19)=$ $6.99, p=.02$, eta-squared $=.27$ ). The number of specific memories recalled from the first person perspective across nine trials of the AMT showed a significant difference between the experimental group $(M=5.08, S D=1.85)$ and control group $(M=7.38, S D=2.13)$ at pre-assessment $(p<.03)$. Post-assessment measurement did not show the hypothesized increase in specific memories recalled from the first person perspective in the experimental group ( $M=4.54$, $S D=2.13, p>.10)$, nor did the control group show any significant difference $(M=7.13, S D=2.23$, $p>$.30). There was a significant between group difference in specific memories recalled from the first person perspective at post-assessment ( $p<.03)$, not accounting for the difference observed at pre-assessment.

Specific Autobiographical Memories recalled from the Third Person Perspective. Results of the repeated measures ANOVA for students' specific memories recalled from the third person perspective as the outcome revealed no significant effect for condition by time (Wilks' $\mathrm{L}=.99$, $F(1,19)=.26, p=.62$, eta-squared $=.01$ ). Time did not show a significant effect (Wilks’ $\mathrm{L}=1.0$, $F(1,19)=.003, p=.96$, eta-squared $<.001)$. There was a marginal main effect by condition $(\mathrm{F}(1,19)=10.0, p=.07$, eta-squared $=.16)$. The number of specific memories recalled from the third person perspective across nine trials of the AMT showed a significant difference between the experimental group $(M=1.62, S D=1.19)$ and control group $(M=.63, S D=.74)$ at preassessment $(p<.04)$. Post-assessment measurement did not show the hypothesized significant 
decrease in specific memories recalled from the third person perspective in the experimental group ( $M=1.54, S D=1.27, p>.40)$, nor did the control group show any significant difference $(M=.75, S D=1.04, p>.36)$. There was no significant between group difference in specific memories recalled from the third person perspective at post-assessment ( $p>$.13).

Vividness of Specific Memories. Results of the repeated measures ANOVA for students' The mean vividness score for specific memories retrieved across nine trials of the AMT as the outcome revealed no significant effect for condition by time (Wilks’ $\mathrm{L}=.95, F(1,19)=1.01, p$ $=.33$, eta-squared $=.05)$. Time showed no significant effect (Wilks’ $L=1.0, F(1,19)=.04, p$ $=.86$, eta-squared $=.18)$. There was no main effect by condition $(\mathrm{F}(1,19)=1.27, p=.27$, etasquared $=.06$ ). The mean vividness score for specific memories retrieved across nine trials of the AMT showed no significant difference between the experimental group $(M=3.76, S D=.95)$ and control group $(M=3.09, S D=1.23)$ at pre-assessment $(p>.21)$. Post-assessment measurement did not show the hypothesized significant increase in mean vividness score for specific memories recalled in the experimental group $(M=3.34, S D=.99, p>.15)$, nor did the control group show any significant difference $(M=3.34, S D=.95, p>.27)$. There was no significant between group difference in mean vividness score for specific memories at post-assessment $(p>.99)$.

\section{Correlations}

Correlation analyses demonstrated that an increase in self-reported mindfulness was positively correlated with a reduction in self-reported rumination in the experimental group, though this result failed to pass significance $(r=.40, p<.09)$. Further analyses showed no significant correlation between increased self-reported mindfulness and changes in recalled autobiographical memories in the experimental group, such as increased specific memory retrieval ( $r=.20, p>.25)$, increased vividness ( $r=.20, p>.25)$, increased specific memories recalled 
from first person perspective ( $r=.11, p>.36)$, and decreased specific memories recalled from third person perspective $(r=-.08, p>.39)$.

An increase in working memory capacity was positively correlated with an increase in specific memories recalled from the first person perspective in the experimental group ( $r=.56$, $p<.03)$. Further analyses showed no significant correlation between increased working memory capacity and changes in recalled autobiographical memories in the experimental group, such as increased specific memory retrieval ( $r=.29, p>.18)$, increased vividness ( $r=.10, p>.37)$, and decreased specific memories recalled from third person perspective ( $r=.45, p>.07)$.

An increase in cognitive flexibility was not significantly correlated to any changes in recalled autobiographical memories in the experimental group, such as increased specific memory retrieval ( $r=.10, p>.37)$, increased vividness ( $r=.08, p>.39)$, increased specific memories recalled from first person perspective $(r=.40, p>.08)$, and decreased specific memories recalled from third person perspective $(r=.38, p>.10)$.

\section{Discussion}

This study examined the effects of mindfulness training on putative indicators of the experiential and narrative modes of self-referential processing. By measuring differences in selfreferential processes through pre- and post-assessments of executive functions, self-report measures of mindfulness and rumination on self, and autobiographical memory retrieval in a group of students receiving mindfulness training, we hypothesized that we would see differences related to increased engagement of the experiential mode of self-referential processing. To test this hypotheses we compared mean scores within subjects across the two assessment periods. To control for possible practice effects, we also compared our experimental group with a control group that received no intervention. 
The experimental group that received mindfulness training did show a significant increase in self-reported mindfulness between pre and post-assessment. The experimental group also significantly decreased in self-reported rumination at post-assessment. The experimental group showed a significant increase in cognitive flexibility, but not working memory capacity, at post-assessment. The experimental group did not recall more specific, first person perspective, or vivid autobiographical memories at post-assessment. Increased mindfulness showed a nonsignificant, but strong correlation with decreased rumination in the mindfulness group only. Self-reported mindfulness did not correlate with any changes in recalled autobiographical memories. The only change in autobiographical memories that significantly correlated with strengthened executive functions appeared in the relationship between working memory capacity and specific memories recalled from the first-person perspective. Significant changes were reported across the two assessment periods for the control group, and must be taken into account. These changes include a decrease in rumination, increase in cognitive flexibility, and increase in autobiographical memory specificity.

\section{Limitations and Future Considerations}

The foremost limitation to this study was the strength of the mindfulness training used on cultivating mindfulness in the students enrolled in the study. Though a significant increase in self-reported mindfulness was observed in the group that received mindfulness training, further analyses did not support this increase as being attributable to the training. As the premise of this study relies on the efficacy of mindfulness training in increasing trainees' engagement in the experiential mode of self-referential processing, a more intense training would be preferable.

Though quantitative methodology was most fitting for our research question, it would be fitting to include some qualitative measures for a better understanding of the subjective 
phenomenological experience of participants. Questions about participants experience in the mindfulness training program, and any reported differences in their subsequent daily habits would not only help us understand individual differences, but also stimulate future hypotheses to explore.

Using only one self-report questionnaire of rumination (the Rumination-Reflection Questionnaire) as a measure of engagement in narrative self-referential processing was another limitation of this study. In the future, multiple measures and self-reports should be used to assess modes of self-referential processing. This limitation does not apply to the use of one self-report questionnaire of mindfulness (the Five Facet Mindfulness Questionnaire) as a measure of engagement in experiential self-referential processing, as mindfulness is synonymous with the experiential mode of self-referential processing, and the questionnaire used is considered an amalgamation of many measures, and highly validated (Baer et al., 2006; Baer et al., 2008). The observed significant increase in cognitive flexibility in both the experimental and control groups may indicate that the Five Point Test is vulnerable to practice effects. It would be wise to include multiple behavioral measures of cognitive flexibility and working memory capacity in order to have better convergent validity on the executive function capacities of participants.

The present study relies on the efficacy of a mindfulness training to induce experiential self-referential processing in participants to such a degree that it may be noticeable during at any given two-hour period in which they were administered a behavioral assessment. This study specifically relies on trait mindfulness, or enduring qualities of mindfulness, rather than state mindfulness, which can be induced with instructions at the beginning of an assessment period. Future studies should explore how both trait and state mindfulness induction concurrently, or differentially, affect outcomes on behavioral measures like the ones used this study. 
Finally, as this study was coordinated around a mindfulness training given during the course of a single college quarter, we cannot be sure how the fluctuating rhythm of schoolrelated demands affected our participants during the pre- and post-assessment. In the future, it might be worth the effort to schedule the post-assessments to occur after finals week, rather than before or during, as was done in the present study. 
References:

Baer, R. A., Smith, G. T., Hopkins, J., Krietemeyer, J., \& Toney, L. (2006). Using self-report assessment methods to explore facets of mindfulness. Assessment, 13, 27-45.

Baer, R. A., Smith, G. T., Lykins. E., Button, D., Krietemeyer, J., Sauer, S., Walsh, E., Duggan, D., \& Williams, J. M. (2008). Construct validity of the five facet mindfulness questionnaire in meditating and nonmeditating samples. Assessment, 15, 329-342.

Conway, M. A., \& Pleydell-Pearce, C. W. (2000). The construction of autobiographical memories in the self-memory system. Psychological Review, 107, 261-288.

Conway, M. A. (2005). Memory and the self. Journal of Memory and Language, 53, 594-628.

Farb, N. A. S., Segal, Z. V., Mayberg, H., Bean, J., McKeon, D., Fatima, Z., \& Anderson, A. K. (2007). Attending to the present: mindfulness meditation reveals distinct neural modes of self-reference. Social Cognitive and Affective Neuroscience, \#, pgs.

Gallagher, S. (2000). Philosophical conceptions of the self: implications for cognitive science. Trends in Cognitive Science, 4, 14-21.

Heeren, A., Van Broeck, N., Philippot P. (2009). The effects of mindfulness on executive processes and autobiographical memory specificity. Behaviour Research and Therapy, 47, 403-409.

James, W. (1890). The principles of psychology. New York: Holt.

Kuyken, W., \& Moulds, M. L. (2009). Remembering as an observer: How is autobiographical memory retrieval vantage perspective linked to depression? Memory, 17(6), 624 - 634.

Macrae, C. N., Moran, J. M., Heatherton, T. F., Banfield, J. F., \& Kelley, W. M. (2004). Medial prefrontal activity predicts memory for self. Cerebral Cortex, 14, 647-654.

McKiernan, K. A., D’Angelo, B. R., Kaufman, J. N., \& Binder, J. R. (2006). Interrupting the "stream of consciousness": an fMRI investigation. Neuroimage, 29, 1185-91.

Nigro, G., \& Neisser, U. (1983). Point of view in personal memories. Cognitive Psychology, 15, 467-482.

Regard, M., Strauss, E., \& Knapp, P. (1982). Children’s production on verbal and non-verbal fluency tasks. Perceptual and Motor Skills, 55, 839-844.

Rogers, T. B., Kuiper, N. A., \& Kirker, W. S. (1977). Self-reference and the encoding of personal information. Journal of Personality and Social Psychology, 35, 677-688.

Sutin, A. R., \& Robins, R. W. (2008). When the "I” looks at the " Me": Autobiographical memory, visual perspective, and the self. Consciousness and Cognition, 17, 1386-1397. 
Symons, C. S., \& Johnson, B. T. (1997). The self-reference effect in memory: A meta-analysis. Psychological Bulletin, 121, 371-394.

Tagini, A., \& Raffone, A. (2010). The 'I' and the 'Me' in self-referential awareness: a neurocognitive hypothesis. Cognitive Processing, 11(1), 9-20.

Trapnell, P. D., \& Campbell, J. D. (1999). Private self-consciousness and the five-factor model of personality: Distinguishing rumination from reflection. Journal of Personality and Social Psychology, 76, 284-304.

Turner, M. L. \& Engle, R. W. (1989). Is working memory capacity task dependent? Journal of Memory and Language, 28, 127-154.

Unsworth, N., Heitz, R. P., Schrock, J. C., \& Engle, R. W. (2005). An automated version of the operation span task. Behavior Research Methods, 37(3), 498-505.

Williams, M., Teasdale, J., Segal, Z., \& Kabat-Zinn, J. (2007). The mindful way through depression. Guilford Press, New York.

Williams, J. M. G., Bardhofer, T., Crane, C., Hermans, D., Raes, F., Watkins, E., \& Dalgleish, T. (2007). Autobiographical memory specificity and emotional disorder. Psychological Bulletin, 133(1), 122-148.

Williams, J. M. G., \& Broadbent, K. (1986). Autobiographical memory in attempted suicide patients. Journal of Abnormal Psychology, 95, 144-149. 